\title{
Benzyl isothiocyanate induces cardiomyocyte proliferation and heart regeneration
}

Akane Sakaguchi ${ }^{1}$, Miwa Kawasaki ${ }^{1}$, Kozue Murata ${ }^{1,3}$, Hidetoshi Masumoto ${ }^{1,2}$ and Wataru Kimura ${ }^{1,}$ $\#$

1: RIKEN Center for Biosystems Dynamics Research, Minatojima-Minamimachi 2-2-3, Chuo-ku, Kobe, Hyogo, Japan 650-0047

2: Department of Cardiovascular Surgery, Graduate School of Medicine, Kyoto University

3: Institute for Advancement of Clinical and Translational Science, Kyoto University Hospital

\#email: wataru.kimura@ riken.jp

running title: BITC induces cardiomyocyte proliferation

Keywords:

Cardiomyocytes; cell cycle; cardiac regeneration: cyclin dependent kinase; myocardial infarction 


\begin{abstract}
Cardiomyocyte proliferation is an evolutionarily conserved mechanism that supports cardiac regeneration in vertebrates. Mammalian cardiomyocytes are arrested from the cell cycle shortly after birth, and therefore mammals lose the ability to regenerate injured myocardium for the rest of their lives. Pharmacological induction of cardiomyocyte proliferation has gained a lot of interest in recent years, as researchers strive to achieve heart tissue regeneration. Here we show that a small chemical, benzyl isothiocyanate (BITC), induced cardiomyocyte proliferation through activation of the cyclin-dependent kinase (CDK) pathway. BITC treatment also allowed heart regeneration in the infarcted neonatal heart, even after the regeneration period in mice. Furthermore, administration of BITC to adult mice in parallel with mild hypoxia $(10 \% \mathrm{O} 2)$ induced cell cycle reentry in the adult heart. Our findings thus suggest that pharmacological activation of the CDK pathway using BITC, concurrently with the activation of hypoxia-related signaling pathways, may be a promising approach to inducing cardiac regeneration in patients with heart disease.
\end{abstract}

\title{
Introduction
}

Mammalian cardiomyocytes have the ability to proliferate from the embryonic stage until the early neonatal stage, with most of them being arrested from the cell cycle shortly after birth ${ }^{1}$. Therefore, adult mammalian heart cannot regenerate myocardial injury, resulting in the fact that ischemic heart disease is the leading cause of death in the world. As such, enhancement of cell cycle reentry for endogenous cardiomyocytes is of great interest for the replacement of the injured fibrotic scar tissue with healthy myocardium ${ }^{2}$. However, despite much attention, pharmacological approaches to the induction of cardiomyocyte proliferation and heart regeneration have yet to be successful.

The cell cycle in cardiomyocytes is precisely regulated by cyclins and cyclin-dependent kinases (CDKs). Postnatal cardiomyocyte cell cycle arrest ${ }^{3,4}$, and subsequent hypertrophy as well as polyploidization, are regulated by other types of cyclin/CDKs ${ }^{5-8}$. Moreover, cyclin/CDKs also regulate the adult cardiomyocyte cell cycle ${ }^{6,9}$. Importantly, overexpression of CDK1, CDK4, cyclin B1, and cyclin D1 promotes cardiomyocyte proliferation in adult heart, and enhances the regeneration of the myocardium after myocardial infarction ${ }^{10}$. Thus, pharmacological activation of cyclin/CDKs potentially stimulates cardiomyocyte proliferation and heart regeneration.

\section{Results and Discussion}

To induce cardiomyocyte proliferation by drug administration, we focused on benzyl isothiocyanate (BITC), which is known to regulate the cell cycle in a dose-dependent manner ${ }^{11}$. First, we subcutaneously administered $20 \mathrm{mg} / \mathrm{kg} /$ day of BITC to neonatal mice from postnatal day 0 (P0) to P6, and examined the number of proliferating cardiomyocytes at P7. The ratio of heart weight per 
body weight was not changed between BITC-treated mice and the control, and the number of phosphorylated histone $\mathrm{H} 3$ ( $\mathrm{pH} 3$ )-positive cardiomyocytes was significantly increased at P7 in the BITC-treated mice compared with control DMSO-treated ones (Fig 1A). Interestingly, cardiomyocyte proliferation did not change upon treatment with 50 or $100 \mathrm{mg} / \mathrm{kg} / \mathrm{day}$ of BITC (data not shown), suggesting that BITC promotes cell proliferation of neonatal cardiomyocytes in a dose-dependent manner. Next, in an attempt to identify the target of BITC, we examined cyclin-dependent kinases (CDKs) because a previous study revealed that CDKs are important for cardiomyocyte proliferation ${ }^{10}$. Phosphorylated CDK1 in myocardial nuclei was increased in the BITC-administered heart at P7 (Fig 1B), indicating that BITC induces the activation of CDK1, which may well contribute to cell cycle progression. Furthermore, after treatment with $50 \mu \mathrm{M}$ BITC, about $70 \%$ of neonatal murine ventricular cardiomyocytes (NMVCs) were positive for $\mathrm{pH} 3$ compared with control (Fig 1C). Similarly, the same treatment with BITC on human iPS cell-derived cardiomyocytes (hiPSC) resulted in a tendency toward increased cell proliferation compared with control, although this enhanced proliferation was not statistically significant, most likely due to a variability among hiPSC batches (Fig 1D). These results indicate that BITC directly induces cell proliferation in cardiomyocytes, rather than via an indirect systemic effect.

Based on these results, we hypothesize that BITC treatment enables heart regeneration after myocardial infarction (MI) through enhanced cardiomyocyte proliferation. To test this hypothesis, we induced MI in P7 mice by permanent ligation of the left anterior descending coronary artery (LAD), and subsequently administered $20 \mathrm{mg} / \mathrm{kg} /$ day of BITC or control DMSO (Fig 2A) from P8 to P14. At P28, the ratio of heart weight per body weight was not changed between BITC-treated mice and the control mice (Fig 2B), on the other hand, BITC-treated mice showed significantly recovered ejection fraction $(\mathrm{EF})$ as well as significantly decreased fibrotic scar compared with DMSO-treated mice (Fig 2C-E), suggesting that BITC administration induces cardiomyocyte proliferation, thereby allowing heart regeneration after $\mathrm{MI}$ in neonatal mice even after the regenerative period. From a therapeutic standpoint, it is important to know whether cell cycle reentry is induced by BITC administration in the adult heart. In order to evaluate the effect of BITC treatment on adult cardiomyocytes, we administered $20 \mathrm{mg} / \mathrm{kg} / \mathrm{day}$ of BITC intraperitoneally to 2-month-old ICR mice for 14 days. In parallel, we placed these mice in a $10 \% \mathrm{O}_{2}$ environment based on findings from a previous study showing that a hypoxic environment aids cell cycle reentry in the adult heart $(\mathrm{Fig} 2 \mathrm{~F})^{12}$. pH3-positive cardiomyocytes were almost absent in the DMSO- or BITC-treated heart under the normal oxygen condition, nor were they present in the DMSO-injected heart in the $10 \% \mathrm{O}_{2}$ condition in consistent with previous findings $(\mathrm{Fig} 2 \mathrm{H})$. In contrast, $\mathrm{pH} 3$-positive cardiomyocytes were significantly increased after BITC administration in the hypoxic environment (Fig 2G, H), indicating that a combination of BITC administration and mild hypoxia exposure induces cell cycle reentry in the adult heart. 
Taken together, our data reveal a beneficial effect of BITC on heart regeneration. BITC treatment enhances cardiomyocyte proliferation through the activation of CDK1 in the neonatal heart. In addition, BITC administration in combination with mild hypoxia in adult mice is sufficient to induce cardiomyocyte cell cycle reentry. The present study suggests that pharmacological activation of the CDK pathway with BITC concurrently with the activation of hypoxia-related signaling pathways may enable researchers to establish a novel strategy to induce cardiac regeneration in patients with heart disease.

\section{Materials and Methods}

Mice

All protocols were approved by the Institutional Animal Care and Use Committee of RIKEN Center for Biosystems Dynamics Research. ICR mice were purchased from CLEA Japan, Inc. and Oriental Yeast Co., Ltd. All experiments were performed on age-matched mice.

\section{Murine model of myocardial infarction}

Induction of anterior wall myocardial infarction was undertaken using a protocol previously described $^{12}$. Briefly, postnatal day (P) 7 mice were anesthetized with hypothermia on ice, and we ligated the left anterior descending coronary artery with polypropylene suture (Ethicon, EP8707H) after thoracotomy. Neonates were warmed on a hot plate $\left(42^{\circ} \mathrm{C}\right)$ after the surgery.

\section{Echocardiography}

Assessment of in vivo cardiac function in conscious mice was undertaken with Affiniti50 with an L15-7io transducer (Phillips) as previously described ${ }^{12}$.

\section{Hypoxia exposure}

Exposure of adult mice to hypoxia was performed as described previously ${ }^{12}$. Briefly, mice were placed in a hypoxia chamber (COY laboratory) and the oxygen concentration was gradually decreased to $10 \%$ over 5 days. After 2 weeks of $10 \% \mathrm{O}_{2}$ exposure in combination with drug administration, oxygen concentration was gradually recovered to $21 \%$ over 5 days.

\section{Human iPS cell derived cardiomyocyte}

The hiPSC lines GCaMP3-253G1 ${ }^{13}$, was used in the present study. The maintenance of hiPSCs and differentiation of cardiovascular cells were conducted in accordance with previous studies ${ }^{14-16}$, with modifications. In brief, hiPSCs were expanded and maintained with StemFit AK02N medium (AJINOMOTO, Tokyo, Japan). At confluence, the cells were dissociated with TrypLE Select (Thermo Fisher Scientific, Waltham, MA, USA), dissolved in $0.5 \mathrm{mM}$ ethylenediaminetetraacetic 
acid in PBS (1:1) and passaged as single cells $\left(5,000-8,000\right.$ cells $\left./ \mathrm{cm}^{2}\right)$ every 5-7 days in AK02N containing iMatrix-511 silk (FUJIFILM Wako Pure Chemical Corp., Osaka, Japan) $(0.125 \mu \mathrm{g} / \mathrm{cm} 2)$ (uncoated laminin fragment) and ROCK inhibitor (Y-27632, $10 \mu \mathrm{M}$, FUJIFILM Wako) ${ }^{17}$. Penicillin-streptomycin (Thermo Fisher Scientific) (10,000 U/mL) (1:100 dilution) was used as required. For cardiovascular cell differentiation, single hiPSCs were seeded onto Matrigel-coated plates (1:60 dilution) at a density of 300,000-400,000 cells $/ \mathrm{cm}^{2}$ in AK02N with Y-27632 (10 $\left.\mu \mathrm{M}\right)$. At confluence, the cells were covered with Matrigel (1:60 dilution in AK02N) one day before induction. We replaced the AK02N medium with RPMI $\square+\square$ B27 medium (RPMI 1640, Thermo Fisher; $2 \mathrm{mM}$ L-glutamine, Thermo Fisher; $1 \times \square$ B27 supplement without insulin, Thermo Fisher) supplemented with $100 \mathrm{ng} / \mathrm{mL}$ Activin A (R\&D, Minneapolis, MN, USA) and 3-5 $\mu$ M CHIR99021 (Tocris Bioscience, Bristol, UK) (differentiation day 0; d0) for $24 \mathrm{~h}$, which was followed with supplementation with $10 \mathrm{ng} / \mathrm{mL}$ bone morphogenetic protein 4 (BMP4; R\&D) and $10 \mathrm{ng} / \mathrm{mL}$ basic fibroblast growth factor (bFGF) (d1) for 4 days without a culture medium change. At d5, the culture medium was replaced with RPMI $\square+\square$ B27+insulin medium (RPMI 1640, Thermo Fisher; $2 \mathrm{mM}$ L-glutamine, Thermo Fisher; $1 \times \square$ B27 supplement with insulin, Thermo Fisher) supplemented with $2.5 \mu$ M IWP4 (Stemgent, Cambridge, MA, USA) and $5 \mu$ M XAV939 (Merck, Kenilworth, NJ, USA). The culture medium was refreshed with RPMI $\square+\square$ B27+insulin medium every other day. Beating cells appeared at d11 to d15. Cardiomyocytes were purified with a specified medium [DMEM without glutamin, glucose and pyruvate, $4 \mathrm{mM}$ L-lactic acid, and $0.1 \%$ BSA (nacalai tesque)] as described previously ${ }^{18}$.

\section{Primary culture}

For cardiomyocyte isolation, 15 hearts were harvested from P1 ICR mice and the ventricles were minced with iris scissors. The pieces of tissue were digested using the enzyme mix from a Neonatal Heart Dissociation Kit, mouse and rat (Miltenyi Biotec 130-098-373) at $37{ }^{\circ} \mathrm{C}$ with gentle pipetting. The suspension was added to 10\% FBS (Thermo 10270106) in DMEM/F12 (Thermo 11320-033) and filtered through a 70- $\mu \mathrm{m}$ cell strainer (Falcon 352350), and then centrifuged (5000g, 5 minutes at $25^{\circ} \mathrm{C}$ ). Precipitated cardiomyocytes were resuspended to $1 \mathrm{ml}$ PBS (Wako 048-29805), treated with $10 \mathrm{ml}$ Red Blood Cell Lysis Solution (Miltenyi 130-094-183) and centrifuged (5000g, 5 minutes at $25{ }^{\circ} \mathrm{C}$ ). The cell pellet was resuspended with culture medium [20\% FBS, 5\% horse serum (gibco 16050-130), 2 mM L-glutamine (gibco 25030-081), 0.1 mM NEAA (gibco 11140-050), 3 $\mathrm{mM}$ sodium pyruvate (gibco 11360-070), $1 \mu \mathrm{g}$ bovine insulin (Sigma I0516) in DMEM/F12] and incubated on a non-coated dish to remove the adhesive cells (e.g. fibrotic cells at $37{ }^{\circ} \mathrm{C}$ for 90 minutes twice. We plated $8 * 10^{5}$ cells/well of isolated cardiomyocytes to fibronectin (cosmo bio SFN01)-coated 4 well chamber slides (Thermo Fisher 154526) and cultured them in a $5 \% \mathrm{CO}_{2}$ incubator. We changed the culture medium 16 hours later to remove debris, and continued 
exchanging the medium once every other day during the culture period.

\section{Drug administration}

Benzyl isothiocyanate (BITC SIGMA 252492) was dissolved in 10\% dimethyl sulfoxide (DMSO WAKO 043-29355)/PBS (nacalai 27575-31), and $20 \mathrm{mg} / \mathrm{kg} / \mathrm{day}$ of BITC was administered to neonatal mice by subcutaneous (s. c.) injection or adult mice by intraperitoneal (IP) injection. Neonatal murine ventricular cardiomyocytes in primary culture and human iPS cell-derived cardiomyocytes (hiPSC) were treated with $50 \mu \mathrm{M}$ BITC (dissolved in $0.1 \%$ DMSO culture medium) for 6 hours.

\section{Immunostaining}

Harvested hearts were fixed with $4 \%$ paraformaldehyde/PBS overnight at $4 \square{ }^{\circ} \mathrm{C}$, and embedded in tissue freezing medium (Genetal Data TFM-C) following replacement with $30 \%$ sucrose/PBS at $4 \square{ }^{\circ} \mathrm{C}$ overnight, and cut to 8 - $\mu \mathrm{m}$ thickness. For immunostaining, antigen retrieval was performed with epitope retrieval solution (IHC World IW-1100-1L) and a steamer (IHC-Tek Epitope Retrieval Streamer Set). Subsequently, tissue sections were blocked with $10 \%$ donkey serum/0.3\% TritonX-100/PBS, and incubated with primary antibodies (cTnT: Thermo Fisher MS295P, 1:100, pH3: Thermo Fisher 06-570, 1:100, pCDK1: abcam ab201008, 1:400) overnight at $4 \square{ }^{\circ} \mathrm{C}$, followed by incubation with corresponding secondary antibodies conjugated to Alexa Fluor 488 or 555 (Invitrogen A21202, A21206, A31570, and A31572), wheat germ agglutinin (50 $\square \mathrm{mg} / \mathrm{ml}$, Thermo Fisher W32466), and DAPI (nacalai, 11034-56) at room temperature. The slides were mounted in antifade mounting medium (nacalai, 12593-64).

\section{Masson's trichrome staining}

TFM-embedded MI heart sections were cut to $10-\mu \mathrm{m}$ thickness and stained with a Trichrome stain kit (ScyTek Laboratories TRM-1-IFU). Protocol is commercially described.

\section{Microscopy}

Images of immunohistochemical, histological, and immunocytochemical samples were taken with Olympus BX53 and Carl Zeiss LSM800 confocal microscope.

\section{Data analyses}

Data are means \pm SEM, the $P$ values were determined with student's $t$ test, and one-way ANOVA was applied for multiple comparison. All scale bars represent $20 \mu \mathrm{m}$.

\section{Acknowledgement}


We thank RIKEN BDR Laboratory for Animal Resources and Genetic Engineering for animal husbandry, and Y. Shiba for providing with a human iPS cell line.

\section{Authors contributions}

A. S. designed and performed the experiments, analyzed the data, and wrote the manuscript. M. K. and K. M. performed human iPS cell-related experiments. H. M. performed and supervised human iPS cell-related experiments. W. K. conceived the study, designed the experiments, analyzed the data and contributed to manuscript preparation.

\section{Conflict of interest}

A. S. has received a collaborative grant from Otsuka Pharmaceutical Co., Ltd as a part of the RBOC funding program Kakehashi.

\section{Figure legends}

Figure 1.

(A) To quantify the number of $\mathrm{pH} 3$-positive cardiomyocytes, BITC- $(20 \mathrm{mg} / \mathrm{kg})$ or DMSO-administered heart sections were stained with pH3 (green) and cardiac troponin T (cTnT, red) antibodies ( $\mathrm{N}=3$ each).

(B) To quantify the number of pCDK1-positive cardiomyocyte nuclei, we used immunofluorescence of pCDK1 (red) and cTnT (green) in BITC-or DMSO-administered heart at $\mathrm{P} 7(\mathrm{~N}=3$ each).

(C) Primary culture of NMVCs were treated with $50 \mu \mathrm{M}$ BITC or DMSO, and the number of $\mathrm{pH} 3$-positive cardiomyocytes was quantified.

(D) hiPSC were treated with $50 \mu \mathrm{M}$ BITC or DMSO, and the number of $\mathrm{pH} 3$-positive cardiomyocytes was quantified.

Figure 2.

(A) Schematic of the experiment. After MI surgery at P7, BITC or DMSO was administered intraperitoneally once a day from P8 to P14

(B) Heart weight normalized with body weight $(\mathrm{HW} / \mathrm{BW})$ was measured at P28.

(C) Left ventricular ejection fraction (EF) was measured by echocardiography blindly at P28.

(D) Representative images of the histological sections of MI heart stained with Masson's trichrome staining are shown.

(E) The quantification of fibrotic area $(\mathrm{N}=3$ each).

(F) Schematic of the experiment. Adult ICR mice were exposed to $10 \% \mathrm{O}_{2}$ or normal conditions and 
injected with BITC or DMSO once a day for two weeks.

(G) A representative image of $\mathrm{pH} 3$-positive cardiomyocytes taken with $\mathrm{z}$-stack imaging with confocal microscopy.

(H) The number of $\mathrm{pH} 3$-positive cardiomyocytes was quantified.

\section{Sources of Funding}

This work was supported by grants from Japan Society for the Promotion of Science (KAKENHI 17H05083, 19K226290, and 20H03680), the PRIME program of the Japan Agency for Medical Research and Development, Takeda Science Foundation, Uehara Memorial Foundation, Japanese Circulation Society, Senri Life Science Foundation, Princess Takamatsu Cancer Research Fund, Mitsubishi Foundation, Bristol-Myers Squibb, Mochida Memorial Foundation, and The Naito Foundation, Toray Science Foundation, a RIKEN CDB/BDR intramural grant to W.K.; and the RBOC funding program Kakehashi to A.S..

\section{Reference List}

1. Soonpaa MH, Kim KK, Pajak L, Franklin M, Field LJ. Cardiomyocyte dna synthesis and binucleation during murine development. Am J Physiol. 1996;271:H2183-2189

2. Sakaguchi A, Kimura W. Metabolic regulation of cardiac regeneration: Roles of hypoxia, energy homeostasis, and mitochondrial dynamics. Curr Opin Genet Dev. $2021 ; 70: 54-60$

3. Tane S, Okayama H, Ikenishi A, Amemiya Y, Nakayama KI, Takeuchi T. Two inhibitory systems and ckis regulate cell cycle exit of mammalian cardiomyocytes after birth. Biochem Biophys Res Commun. 2015;466:147-154

4. Mahmoud AI, Kocabas F, Muralidhar SA, Kimura W, Koura AS, Thet S, Porrello ER, Sadek HA. Meisl regulates postnatal cardiomyocyte cell cycle arrest. Nature. $2013 ; 497: 249-253$

5. Di Stefano V, Giacca M, Capogrossi MC, Crescenzi M, Martelli F. Knockdown of cyclin-dependent kinase inhibitors induces cardiomyocyte re-entry in the cell cycle. J Biol Chem. 2011;286:8644-8654

6. Tane S, Kubota M, Okayama H, Ikenishi A, Yoshitome S, Iwamoto N, Satoh Y, Kusakabe A, Ogawa S, Kanai A, Molkentin JD, Nakamura K, Ohbayashi T, Takeuchi T. Repression of cyclin d1 expression is necessary for the maintenance of cell cycle exit in adult mammalian cardiomyocytes. J Biol Chem. 2014;289:18033-18044

7. Tamamori-Adachi M, Ito H, Nobori K, Hayashida K, Kawauchi J, Adachi S, Ikeda MA, Kitajima S. Expression of cyclin $\mathrm{d} 1$ and cdk4 causes hypertrophic growth of 
cardiomyocytes in culture: A possible implication for cardiac hypertrophy. Biochem Biophys Res Commun. 2002;296:274-280

8. Liu Z, Yue S, Chen X, Kubin T, Braun T. Regulation of cardiomyocyte polyploidy and multinucleation by cycling1. Circ Res. 2010;106:1498-1506

9. Bickne11 KA, Coxon CH, Brooks G. Forced expression of the cyclin b1-cdc2 complex induces proliferation in adult rat cardiomyocytes. Biochem J. 2004;382:411-416

10. Mohamed TMA, Ang YS, Radzinsky E, Zhou P, Huang Y, Elfenbein A, Foley A, Magnitsky S, Srivastava D. Regulation of cell cycle to stimulate adult cardiomyocyte proliferation and cardiac regeneration. Cell. 2018;173:104-116. el12

11. Akagi K, Sano M, Ogawa K, Hirose M, Goshima H, Shirai T. Involvement of toxicity as an early event in urinary bladder carcinogenesis induced by phenethyl isothiocyanate, benzyl isothiocyanate, and analogues in f344 rats. Toxicol Pathol. $2003 ; 31: 388-396$

12. Nakada Y, Canseco DC, Thet S, Abdisalaam S, Asaithamby A, Santos CX, Shah AM, Zhang H, Faber JE, Kinter MT, Szweda LI, Xing C, Hu Z, Deberardinis RJ, Schiattarella G, Hill JA, Oz 0, Lu Z, Zhang CC, Kimura W, Sadek HA. Hypoxia induces heart regeneration in adult mice. Nature. 2017;541:222-227

13. Shiba Y, Fernandes S, Zhu WZ, Filice D, Muskheli V, Kim J, Palpant NJ, Gantz J, Moyes KW, Reinecke H, Van Biber B, Dardas T, Mignone JL, Izawa A, Hanna R, Viswanathan M, Gold JD, Kotlikoff MI, Sarvazyan N, Kay MW, Murry CE, Laflamme MA. Human es-cel1-derived cardiomyocytes electrically couple and suppress arrhythmias in injured hearts. Nature. 2012;489:322-325

14. Masumoto H, Nakane T, Tinney JP, Yuan F, Ye F, Kowalski WJ, Minakata K, Sakata R, Yamashita JK, Keller BB. The myocardial regenerative potential of three-dimensional engineered cardiac tissues composed of multiple human ips ce11-derived cardiovascular cell lineages. Sci Rep. 2016;6:29933

15. Nakane T, Masumoto H, Tinney JP, Yuan F, Kowalski WJ, Ye F, LeBlanc AJ, Sakata R, Yamashita JK, Keller BB. Impact of cell composition and geometry on human induced pluripotent stem cells-derived engineered cardiac tissue. Sci Rep. 2017;7:45641

16. Abulaiti M, Yalikun Y, Murata K, Sato A, Sami MM, Sasaki Y, Fujiwara Y, Minatoya K, Shiba Y, Tanaka Y, Masumoto H. Establishment of a heart-on-a-chip microdevice based on human ips cells for the evaluation of human heart tissue function. Sci Rep. 2020;10:19201

17. Miyazaki T, Isobe T, Nakatsuji N, Suemori H. Efficient adhesion culture of human pluripotent stem cells using laminin fragments in an uncoated manner. Sci Rep. $2017 ; 7: 41165$ 
18. Tohyama S, Hattori F, Sano M, Hishiki T, Nagahata Y, Matsuura T, Hashimoto H, Suzuki T, Yamashita H, Satoh Y, Egashira T, Seki T, Muraoka N, Yamakawa H, Ohgino Y, Tanaka T, Yoichi M, Yuasa S, Murata M, Suematsu M, Fukuda K. Distinct metabolic flow enables large-scale purification of mouse and human pluripotent stem cell-derived cardiomyocytes. Cell Stem Cell. 2013;12:127-137 
Figure 1

(which was not certified by peer review) is the author/funder. All rights reserved. No reuse allowed without permission.

(A)

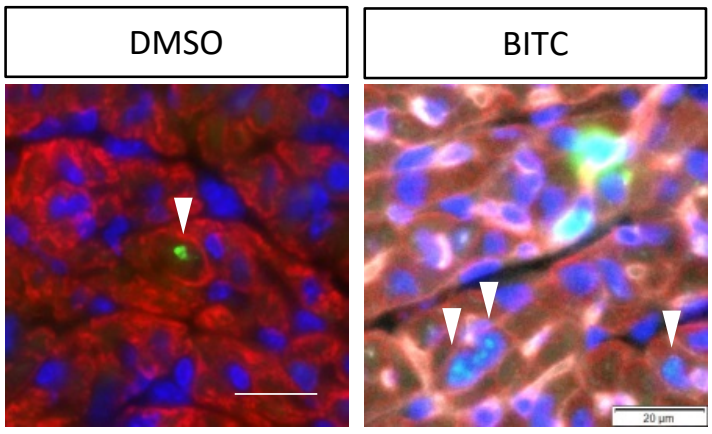

pH3, cTnT, WGA, DAPI

(B)

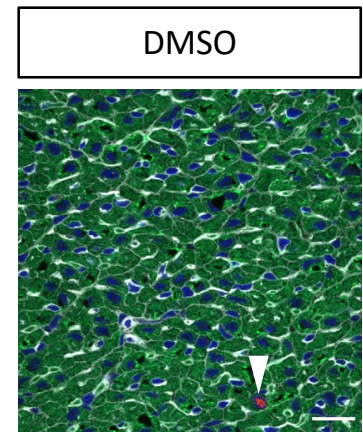

pCDK1, cTnT, WGA, DAPI

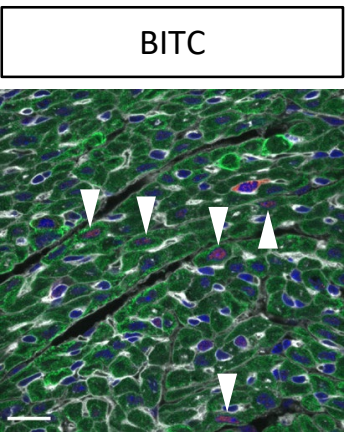

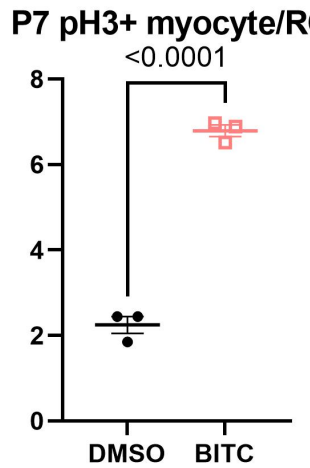

pCDK1+ myocyte/ROI

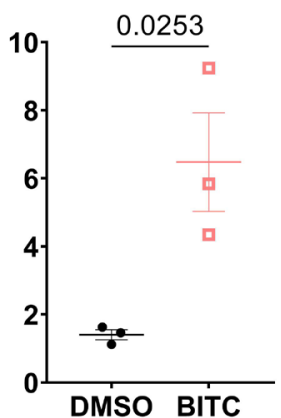

(C)
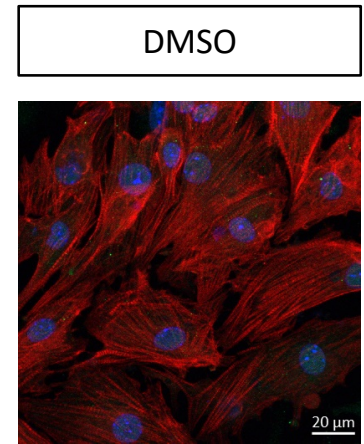

cTnT, pH3, DAPI
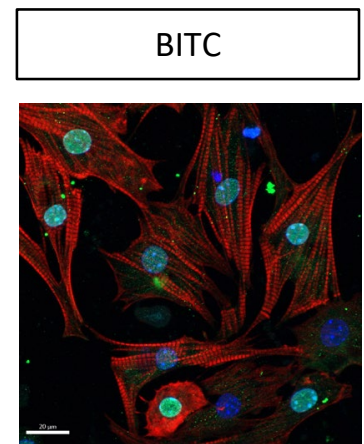

(D)
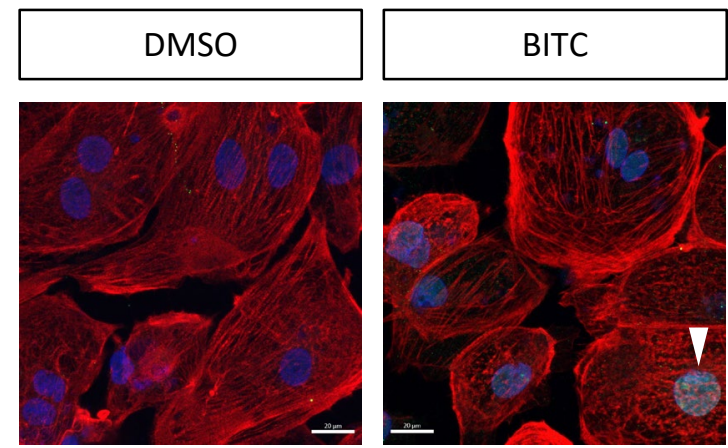

cTnT, pH3, DAPI
pH3+ myocyte/ROI (\%)

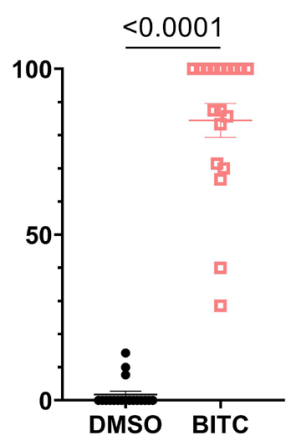

pH3+ hiPS-myocyte (\%)

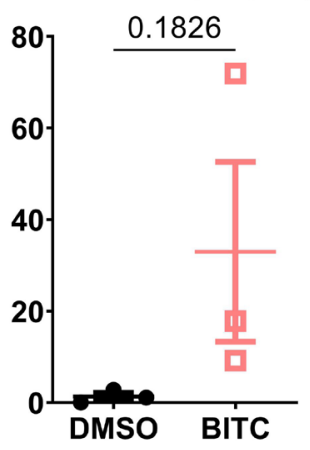

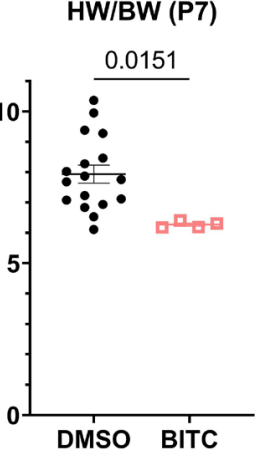


(A)

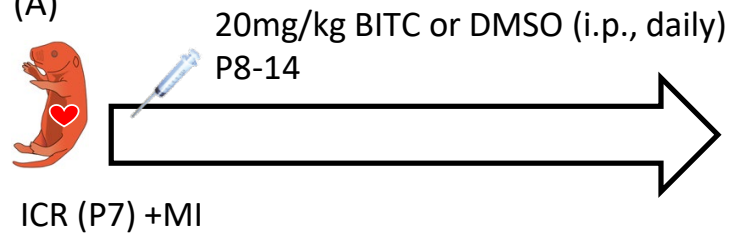

(B)

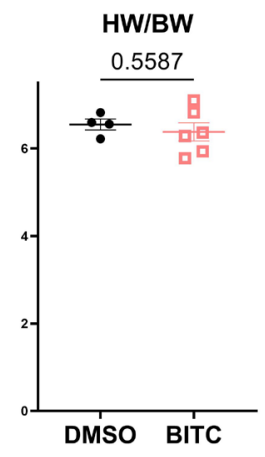

(C)

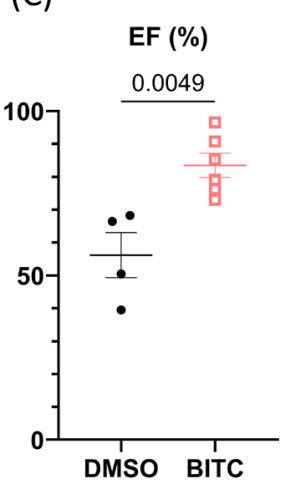

(D)

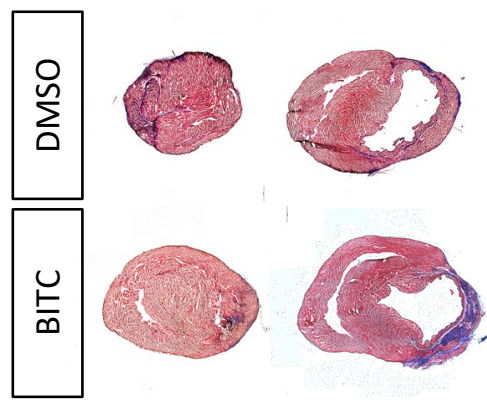

(F)

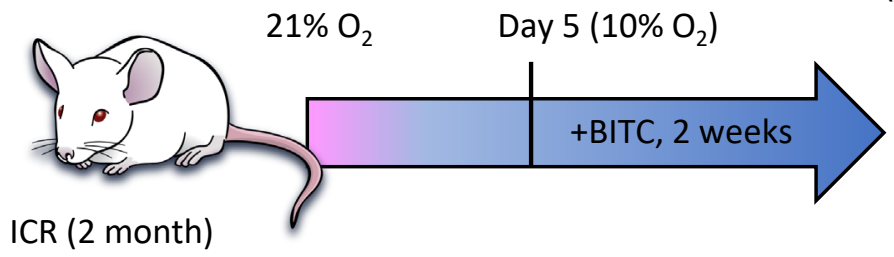

(G)

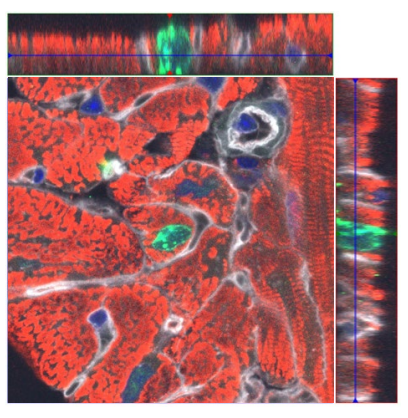

pH3, cTnT, WGA, DAPI
(E)

fibrotic are/secton (\%)

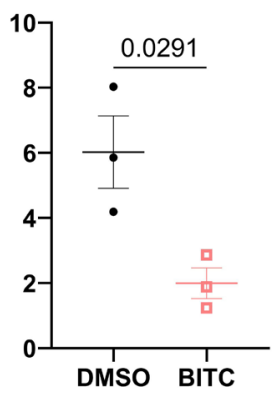

(H)

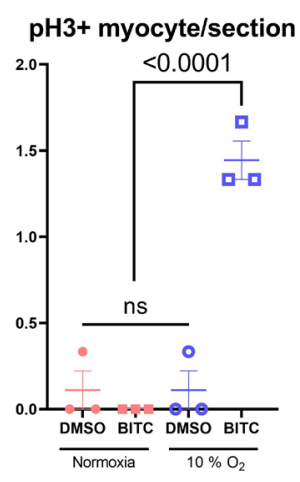

